\title{
Precession dynamics in NiFe thin films, induced by short magnetic in-plane field pulses generated by a photoconductive switch
}

\author{
Th. Gerrits, H.A.M. van den Berg*, J. Hohlfeld, O. Gielkens, K.J. Veenstra, K.Bal and Th. Rasing \\ Research Institute for Materials, Toernooiveld 1, 6525 ED Nijmegen, The Netherlands \\ *Siemens AG, ZT MF 1, Paul-Gossen-Strasse 100, 91034 Erlangen, Germany
}

(Received Oct. 9, 2000; Accepted Jan. 15, 2001)

\begin{abstract}
Short magnetic in-plane field pulses were used to pull the magnetic spin system of a NiFe film out of its equilibrium state and to initiate coherent magnetisation dynamics. This field induced dynamics was investigated by means of a time-resolved pump-probe experiment, measuring the linear Kerr effect with a balanced photodiode scheme. Precession frequencies of several $\mathrm{GHz}$ and relaxation-times in the nanosecond range were observed. Emphasis is on the generation of the short magnetic in-plane field pulses. They were generated using an ultrafast photoconductive switch based on GaAs and launched by a waveguide to the sample. The waveguide and the photoswitch were designed to yield as large as possible magnetic field strengths. The field pulses used in this work had a rise time of $10-60 \mathrm{ps}$, a decay time of $500-700 \mathrm{ps}$ and a maximum field strength of $90 \mathrm{e}$.
\end{abstract}

Key words: photoconductive switch, coplanar waveguide, precession dynamics, short magnetic in-plane field pulses, MRAM, time-resolved Magneto Optical Kerr Effect, magnetic recording, soft materials

\section{Introduction}

The study of spin dynamics in magnetic media has gained more and more interest in the last few years, as the writing speed of data on magnetic media is increasing rapidly. As soon as writing times of less than a nanosecond are reached, spin dynamic effects play the dominant role in the writing process. The writing process pulls the magnetic spin system out of its equilibrium state. The relaxation process to its new equilibrium state is determined by the magnetic spin dynamics of the media. The study of the dynamics on ultra short time scales can best be done by using magnetic field pulses, which are shorter in time than the relaxation process of the system. The great interest of using in-plane field pulses to study the dynamics of a ferromagnetic system lies in its impact on the writing process of magnetic RAMs (MRAM), for which in-plane field pulses of optimised shape are required. The design of our device is done in a way that we in principle can generate pulses of arbitrary shape.

Experiments have already been performed, using in-plane pulses. However, the field pulses in the experiments reported so far were generated by pulse generators ${ }^{1)}$ and only the rise times were shorter than the relaxation of the system. Other experiments, also using pulses generated by a GaAs switch, initiated the dynamics by a polar field pulse ${ }^{2)}$ in a single coil.

Here we report investigations on the dynamics of a ferromagnetic system following excitation by $10-60$ ps rise time,
$500-700$ ps decay time magnetic in-plane field pulses which were in total much shorter than the magnetic relaxation time. Thus the magnetic response of the system at large pump-probe delays was solely governed by the magnetic properties of the sample. The response was probed by a time-resolved Magneto Optical Kerr Effect experiment in combination with the balancing photodiodes technique ${ }^{3)}$. We will show that our experimental approach is best suited to study the spin dynamics of a weak ferromagnetic system.

\subsection{Device}

\section{Technique}

To generate short magnetic field pulses, we use a GaAs photodiode in combination with a coplanar waveguide. As GaAs is a direct semiconductor, light with an energy above the band gap will excite carriers to the conduction band and the creation of carriers is directly correlated to the profile of the laser pulse.

The device consists of two copper electrodes structured into a coplanar waveguide. A bias voltage is applied to the copper electrodes. While illuminating the gap between the electrodes, an electrical pulse is generated and travels through the waveguide. The rise time of the electrical pulse at the gap only depends on the capacitance, the inductance and the resistance of the gap ${ }^{4}$. While travelling through the waveguide, dispersion and attenuation of the terahertz pulse is observed ${ }^{5}$. The decay time of the pulse is determined by the carrier lifetime of the material. Undoped GaAs has typical lifetimes of $100 \mathrm{ps}-500 \mathrm{ps}$, whereas low temperature grown GaAs may have lifetimes of a few picoseconds?

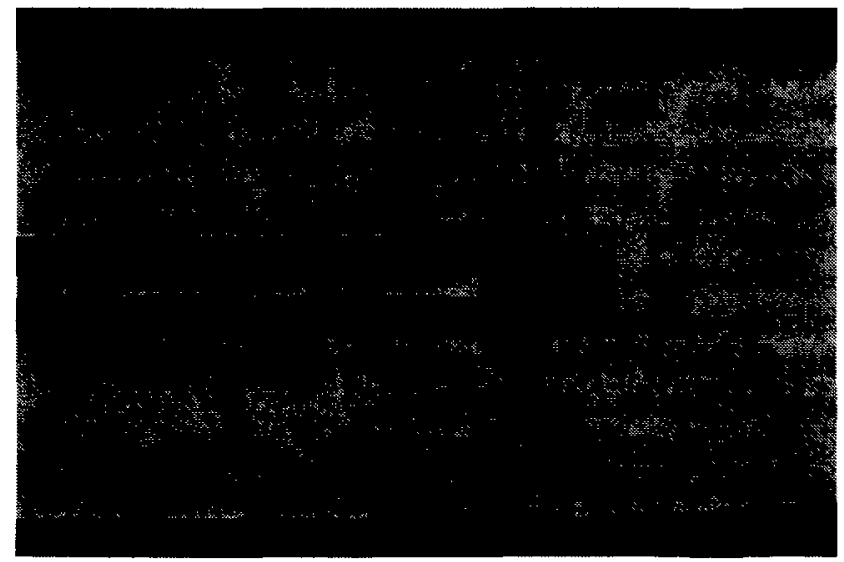

\section{Figure 1}

A photograph of the photoswitches. Between the finger structure, another light electrode can be seen. This is a thin copper electrode that should prevent shadow effects form the larger electrodes 


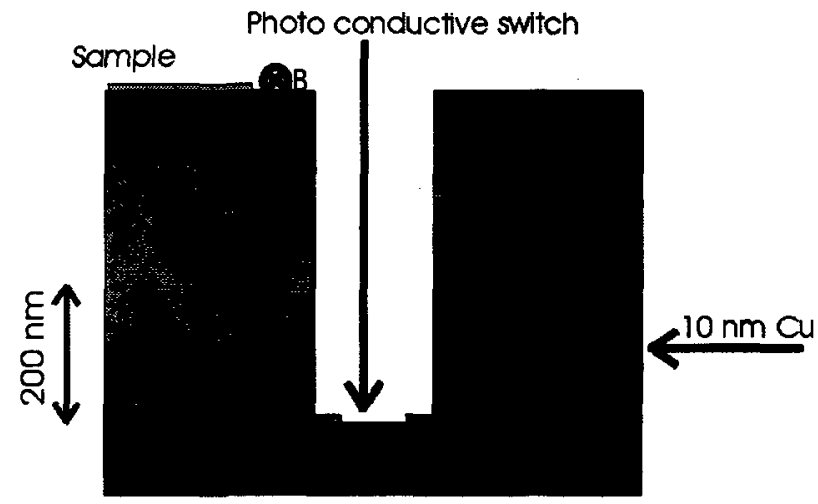

Figure 2

A cross section of the device. The 100 fs laser beam pumps the gap with the finger electrodes. A current pulse is coupled into the waveguide and passes the sample, while inducing an in-plane magnetic field. The $10 \mathrm{~nm}$ copper layer is used to overcome shadow effects due to the thick electrodes

Figure 1 shows a photograph of the Auston switch. It shows a finger structure, which enlarges the area for the excitation of carriers and thus the total current. The gap between the electrodes is $15 \mu \mathrm{m}$. As the pump laser beam hits the device under a certain angle, the electrodes would give rise to some dark area within the photoswitch. This would result in a large resistance due to the dark area within the photoswitch and would decrease the generated current. Our finger structure is designed in a way that at the edges of the thick electrodes, $10 \mathrm{~nm}$ thin electrodes are present, which are separated $5 \mu \mathrm{m}$ from each other. As the thickness of the electrodes is chosen to be smaller than the skin depth for the incoming laser beam, light can travel through and excite carriers beneath. So no resistance due to dark areas within the switch is present. This technique makes it possible to change the angle of incidence of the pump beam without changing the resistance of the photoswitch.

Figure 2 shows a cross section of our device. The top layer consist of a $16 \mathrm{~nm} \mathrm{NiFe} \mathrm{(81:19)} \mathrm{film,} \mathrm{sputtered} \mathrm{by} \mathrm{magnetron}$ sputtering. The lateral dimensions of the NiFe film are $10 \times 20$ $\mu \mathrm{m}$ and it only lies on top of the signal line (the middle electrode of the waveguide). This film is covered with a $3 \mathrm{~nm} \mathrm{Cr}$ layer to prevent corrosion. Close to the surface of the electrode, the magnetic field due to a current pulse is homogeneous in-plane. For insulation of the NiFe film from the electrodes, we used a 100 $\mathrm{nm}$ thin layer of $\mathrm{SiO}_{2}$. This thickness is small compared to the dimensions of the signal line, so that we can ensure a homogeneous in-plane field over the probed area of the NiFe film. The copper electrode consists of a thick $250 \mathrm{~mm}$ layer and a thin $10 \mathrm{~nm}$ layer. Beneath the copper electrodes, another insulating layer of $\mathrm{SiO}_{2}$ is deposited, that ensures that the electrodes are insulated from each other. No leakage current can flow from the signal line to the waveguide ground flats through the wafer. Only the photoswitches are kept free. As substrate we used undoped GaAs.

Figure 3 shows a photograph of the complete waveguide structure. The two photoswitches with dimensions of $65 \times 65 \mu \mathrm{m}$ (white arrows) are rather big in comparison to the signal line, which is only $10 \mu \mathrm{m}$ wide (black arrow). The photoswitches can be used as one switch only, or can be used in a pump-pump-probe experiment, where the voltage on the electrodes can be arbitrary.
By means of such an experiment it is possible to apply opposite voltages to the electrodes. By choosing a time delay between two pump pulses, one can in principle shorten the pulse generated first $^{8}$. Another possibility is to apply the same voltages and so by means of two pump pulses make the field pulse longer. With this technique it is in principle possible to produce arbitrary pulse shapes, by choosing the right photoconductive material and bias voltages. Here we restrict ourselves to the excitation of a single switch only.

In onder to generate large magnetic fields on the signal line, large currents and small signal lines are needed, as the total current I generated in the photoswitch and sent through the waveguide depends on the carrier density times the area of the photoswitch, whereas the magnetic field $\mathrm{H}$ close to the surface of the signal line is proportional to the current density in the signal line: $\mathrm{H}=\mathrm{I} / 2 \mathrm{w}$.

For the design of the waveguide we used a model ${ }^{5,7}$, which describes the propagation of the current pulse on the signal line. It describes the attenuation and dispersion due to the surface impedance of a coplanar stripline, including the dielectrics, surrounding it. According to this model we chose the dimensions of the signal line in a way that $0.01-1 \mathrm{THz}$ pulses will have lowest possible attenuation and dispersion. The width of the signal line was chosen to be $10 \mu \mathrm{m}$. The spacing between the signal line and the ground flats is also $10 \mu \mathrm{m}$. In order to concentrate the current onto the signal line, the tapering was designed by keeping the ratio between the middle line and the spacing constant, as that should not change the impedance of the waveguide ${ }^{5,7}$. A change in impedance would cause reflections on the signal line, which will broaden the current pulse and lower the maximum obtainable field.

\subsection{The Experimental Setup}

The experimental setup is shown figure 4. We use a $100 \mathrm{fs}$ pulsed $\mathrm{Ti}$ : Sapphire laser for our measurements on the NiFe thin films. The laser is sent to a BK7 beam splitter, which divides the beam into two parts: a pump beam and a probe beam. The pump beam is used to excite the photoswitch. After the beam splitter, the pump beam is brought onto a retro reflector, mounted on a 50 $\mathrm{mm}$ long translation stage. This translation stage has a step width of 1 micron, which corresponds to a pump beam time-delay of $\approx$ $7 \mathrm{fs}$ in air. The translation stage is mounted onto a

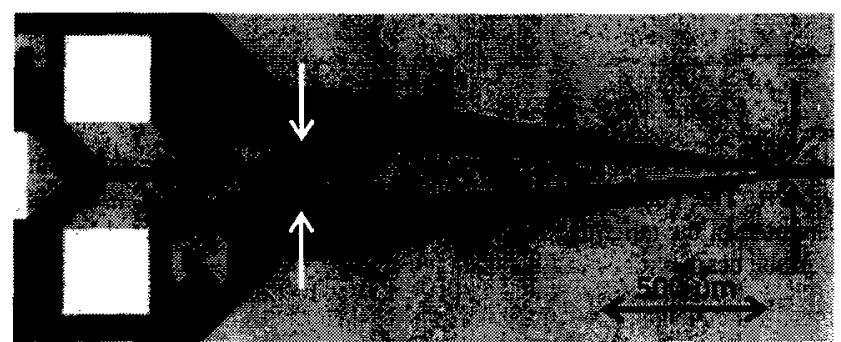

Figure 3

A photograph of the waveguide, used in our experiment. The tapering concentrates the current onto the $10 \mu \mathrm{m}$ signal line. At the end of the tapering, the thin $10 \times 20 \mu \mathrm{m}$ film is placed. Around the signal line, two big ground flats are placed as a waveguide. 


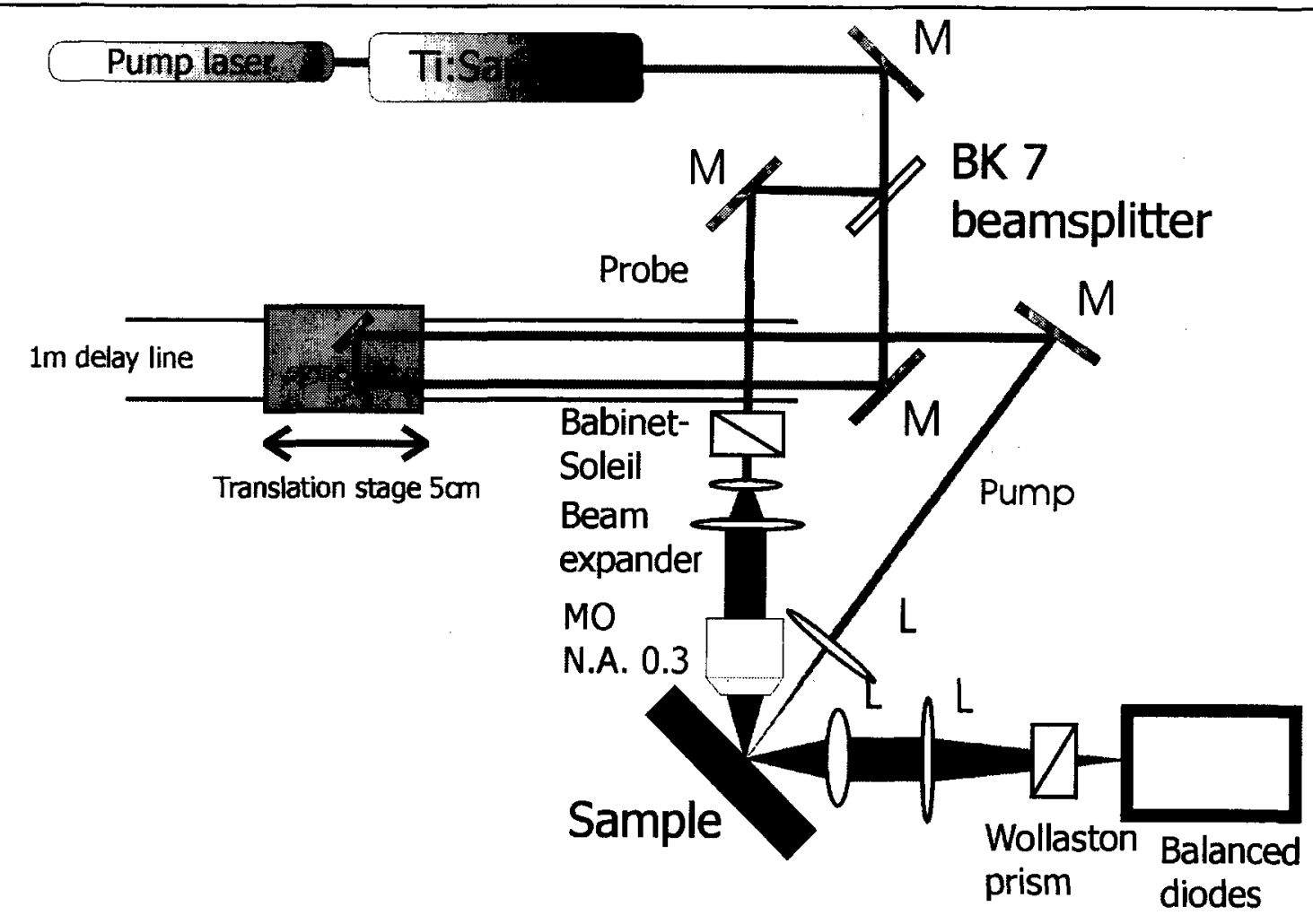

Figure 4

The experimental setup for a time resolved pump-probe-MOKE experiment

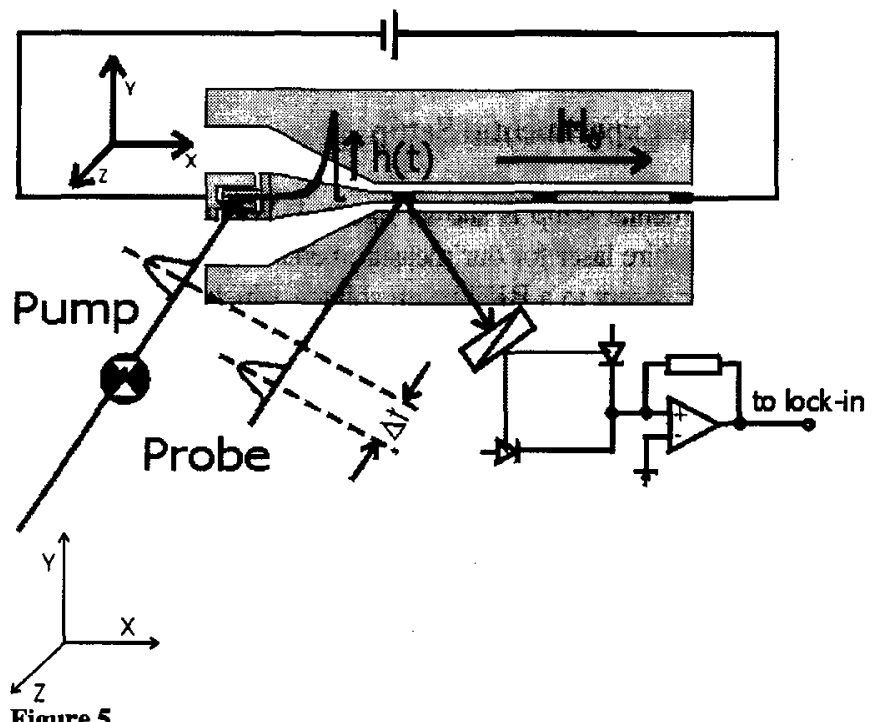

Scheme of the waveguide with the signal line and the magnetic thin film lying on it. The $100 \mathrm{fs}$ pump pulse generates the current pulse, which is concentrated in the tapering. The response of the system is measured by the 100 fs probe beam.
$1 \mathrm{~m}$ long delay line. We can move the translation stage on the delay line with an accuracy of $0.5 \mathrm{~mm} \approx 3.3 \mathrm{ps}$. The combination

of a long delay line and a short translation stage with small steps, allows us to measure both nanosecond and femtosecond dynamics. Returning from the retro reflector the pump beam is directed by another mirror to the device, where it is focused at normal incidence onto the photoswitch.

The probe beam travels to a Babinet-Soleil compensator, which is used for changing the polarization of the beam. After the

Babinet-Soleil compensator, the probe beam is expanded by a factor five and subsequently focussed by a long working distance microscope objective (N.A. 0.3 ) to a spot-size of $5 \mu \mathrm{m}$ on the $\mathrm{NiFe}$ film. The use of a long working distance objective was necessary to avoid screening of the pump beam.

The whole setup is designed in a way that we can probe the magnetization under an angle of incidence of $45^{\circ}$ as well as in normal incidence, so that we can probe the polar effect in the $0^{\circ}$ configuration only and probe the longitudinal + polar effect with the $45^{\circ}$ configuration. The $45^{\circ}$ configuration makes it also possible to probe by means of $\mathrm{MSHG}^{10}$, where the transversal component of the magnetisation can be measured. Figure 5 shows a schematic drawing of the waveguide. A longitudinal magnetic bias field is applied to the sample. The pump beam is chopped and activates the photoswitch. With the probe beam we measured the linear MOKE signal by means of the balancing diodes and lock-in technique ${ }^{3)}$. While delaying the pump beam, we are able 
to get the temporal information of the $\mathrm{NiFe}$ system due to the short magnetic field pulse.

\section{Precession Dynamics}

The spin dynamics of the ferromagnetic NiFe system can well be described in terms of the Landau-Lifshitz equation with the Gilbert damping term:

$$
\mathrm{d} \mathbf{M} / \mathrm{dt}=\gamma(\mathbf{M} \times \mathbf{H})-\alpha(\mathbf{M} \times \mathrm{d} \mathbf{M} / \mathrm{dt})
$$

The value of $\gamma$ is given by $-g \mu_{\mathrm{B}} / \mathrm{h}$, where $\mu_{\mathrm{B}}$ and $\mathrm{g}$ are the Bohr magneton and the spectroscopic splitting factor respectively. The Gilbert damping of the system is represented by $\alpha$. In our fits to the measured precessions, we took $\gamma=17.6 \cdot 10^{6}(\mathrm{Gs})^{-1}$ and estimated $\alpha=0.008$. In eqn. $1 \mathbf{H}$ denotes the total field within the system:

$$
\mathbf{H}=\mathbf{H}_{\mathrm{ext}}+\mathbf{H}_{\mathrm{s}}
$$

where $H_{e x t}$ is given by the applied bias field $\left(H_{0}\right)$ and the field pulse $h(t) . H_{e x t}=\left[H_{0}, h(t), 0\right]$ and $\mathbf{H}_{s}$ represents the shape anisotropy field of the film. Figure 6 shows the precession of the system while the field pulse passes the sample. The left part of figure 6 shows the magnetisation in its equilibrium state along the $x$ axis before the field pulse arrives. As the field pulse passes the sample, the magnetisation is pulled out of plane ( $z$ direction); the precession is started. While passing the sample, the field pulse produces a new centre of precession for the system, given by the vector sum of the bias field and the field pulse. Also the precession frequency will increase, as the frequency depends on the strength of the bias field, which is now the sum of $\mathbf{H}_{0}$ and $\mathbf{h}(\mathrm{t})$. When the field pulse has passed the sample, the period of precession is determined by the applied bias field and the film anisotropy only.

In general the precession frequency $\omega$ is given by the determinant of the coupled system of differential equations. The exact calculation of $\omega$ is rather complicated since the time dependent field pulse has to be included. For large bias fields, however its value is given in good approximation by assuming that $M_{x}$ is constant and equal to the saturation magnetisation $M_{s}$. One obtains:

$$
\omega^{2}=\gamma^{2}\left[H_{0}+4 \pi M_{s}+H_{s x}\right]\left[H_{0}+H_{s x}\right]
$$

where $\mathrm{H}_{\mathrm{sx}}$ is representing the sum of the induced and the shape anisotropy field of our thin film by an effective field in the $x$ direction. A change of this effective field over the thin film area will influence the precession frequency. We assumed an easy in-plane anisotropy and therefore the factor $4 \pi \mathrm{M}_{\mathrm{s}}$ originates from the magnetostatic field in the $z$ direction of the thin film. Note that this expression is only valid if the field due to the pulse is small compared to the applied bias field. From eqn. 3 it can easily be seen that the anisotropy field of the film element can be determined for $\mathrm{H}_{0}=0$.
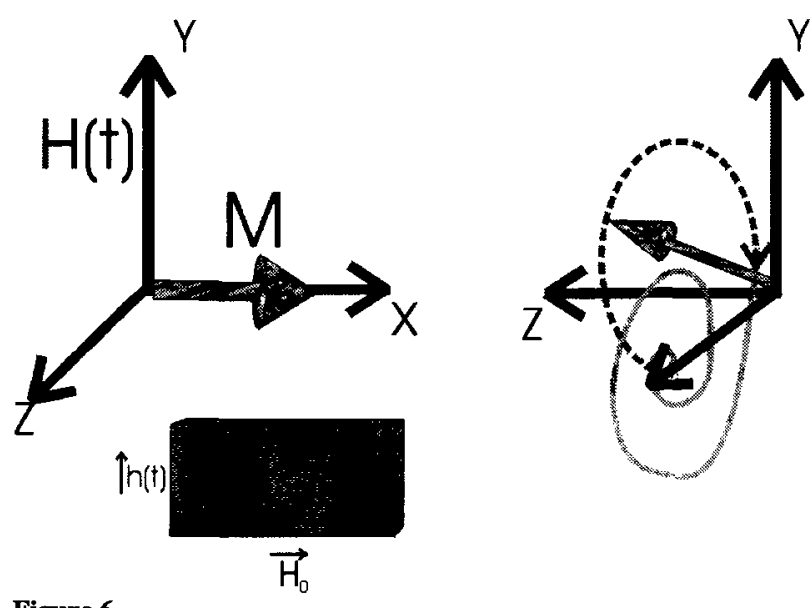

Figure 6

Schematic behaviour of the precession during the magnetic field pulse. In the left part of the figure, the magnetisation is parallel to the applied bias field $\mathbf{H}_{0}$. The right part shows the precession while the pulse passes the sample. The centre axis is tilted into the direction of the field pulse. The precession gets faster (dashed line). As the field pulse decays, the system finds its equilibrium along the $\mathrm{x}$ axis of the system (solid line). The thin film plane lies in the $x-y$ plane.

\section{Results}

Figure 7 shows the time resolved magneto optical response of the NiFe (81:19) film in the $45^{\circ}$ configuration. We can describe this dynamics by solving the LLG equation. Equation 1 shows that the frequency of precession depends also on the $y$ component of the magnetic field pulse. Thus the magnetic field pulses will act as an additional bias field applied, as the system will find a new centre axis of precession". Figure 6 shows the effect of the new centre axis due to the field pulse.

A stronger effective bias field causes an increase of the precession frequency. This can be seen in fig. 7: The precession frequency decreases until it asymptotically reaches a lowest value with the $x$ axis of the system as its centre axis of precession with a frequency determined by the bias field and the anisotropy of the film element(c.f. eqn. 3). Our thin film is prepared by magnetron sputtering in a way that we induced an anisotropy along the long axis of the elements.

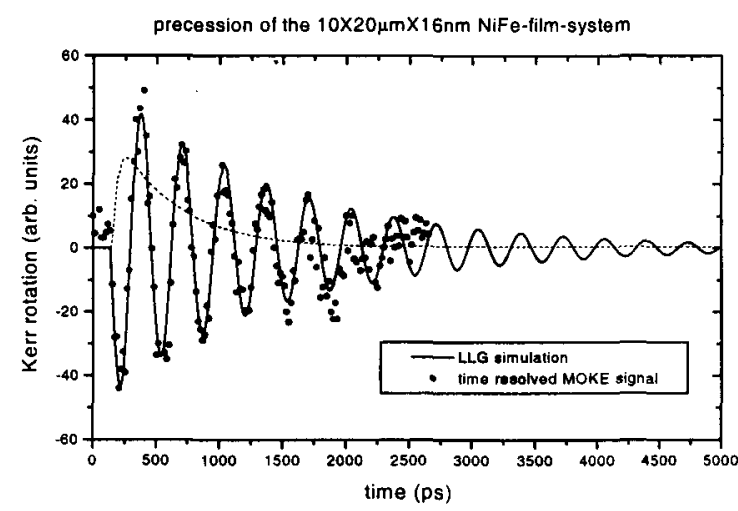

Figure 7

Precession of the ferromagnetic NiFe system as measured by a time-resolved pump-probe MOKE experiment in the $45^{\circ}$ configuration. For this measurement the bias field was 940 . The solid line shows the LLG simulation for the given system. The dashed line shows the estimated magnetic field pulse. 


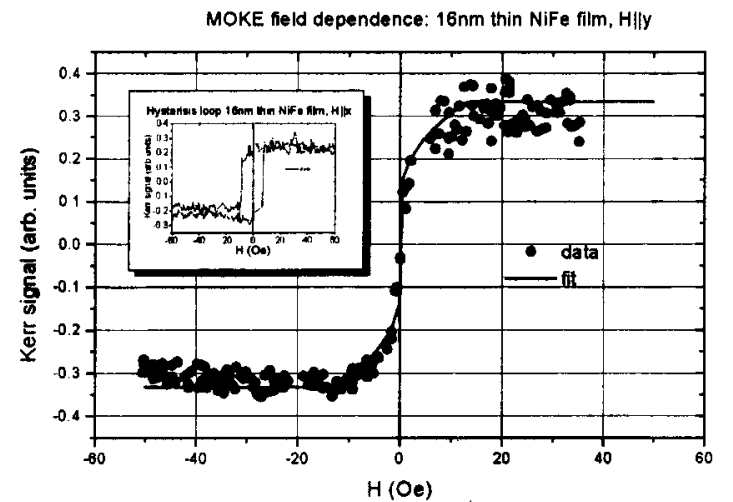

Figure 8

MOKE hysteresis loops for the $10 \times 20 \mu \mathrm{m}$ NiFe film. The circles represent a measurement along $y$-axis of the system. The anisotropy easy axis is found to be in the $x$ direction of the thin film. The fit to the data (solid line) was obtained by a second order anisotropy. The inset shows the hysteresis loop along the easy axis of the thin film.

To quantify this, measurements have been done in order to determine the parameters necessary for the simulation. The determination of the anisotropy of the film is of primary interest, as it influences the torque experienced by the dipoles during the precession.

Figure 8 shows the hysteresis loop for the NiFe thin film, measured perpendicular to the anisotropy easy axis. We can fit this loop, using the Stoner-Wohlfarth model for an uni-axial second order anisotropy. The anisotropy energy of the system is given by:

$$
E=-K_{1} \cos ^{2} \theta-K_{2} \cos ^{4} \theta
$$

and the effective anisotropy field $\mathrm{H}_{\mathrm{s}}$ in the $x$ direction of the system is:

$$
\mathrm{H}_{\mathrm{s}}=\mathbf{M}_{\mathrm{s}}^{-1} \partial \mathrm{E} / \partial \cos \theta
$$

where $\theta$ denotes the angle between the magnetisation and the easy anisotropy axis $(x)$ of the film and $\mathrm{K}_{1}, \mathrm{~K}_{2}$ represent the

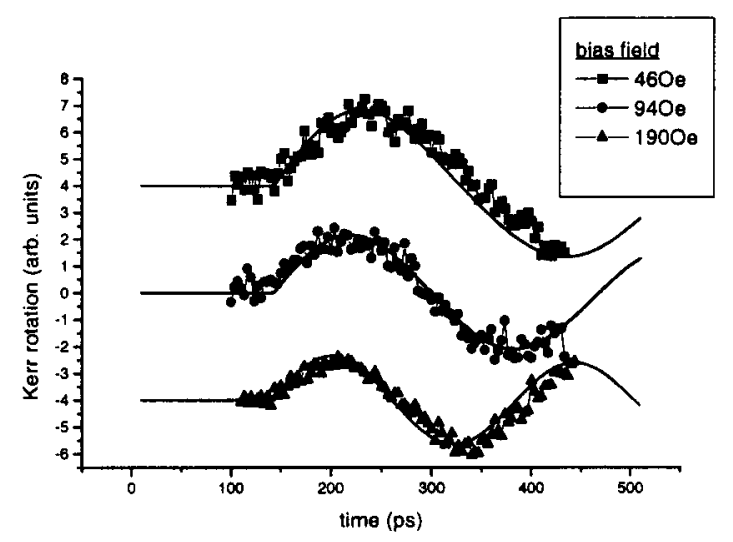

Figure 9

Start of precession for different bias fields. From this data we were able to estimate the rise time of the magnetic field pulse. The solid lines represent the simulated precession due to a field pulse with 30ps rise time

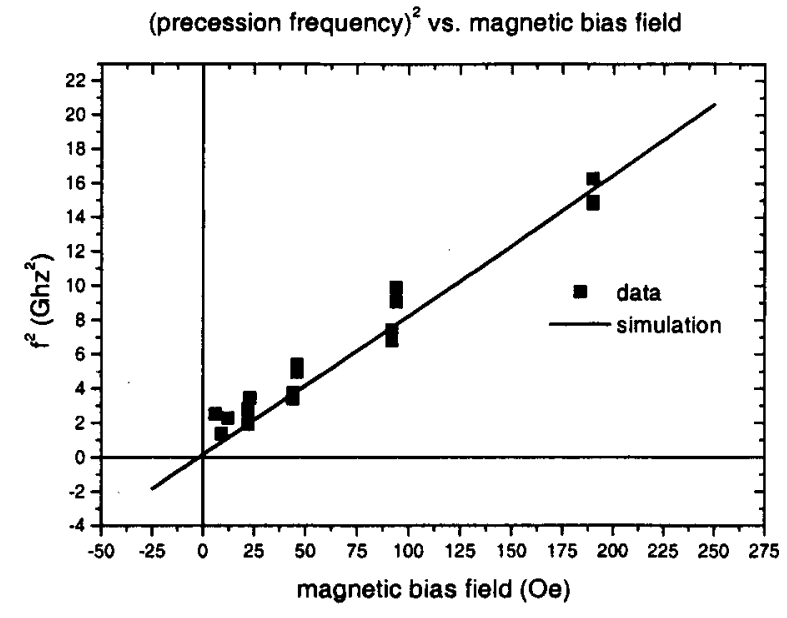

Figure 10

Square of the measured precession frequency versus the applied bias field. The solid line shows the simulation from eqn. (3). From the measured data one can see that at low bias fields, the precession frequency deviates from the ideal linear behaviour. This may be due to remains of the field pulse, which increases the precession frequency.

strength of anisotropy. Note that these constants represent both the magnetostatic and induced anisotropy. By fitting the hysteresis loop in fig.8, we determined: $\mathrm{K}_{1}=5.2 \cdot 10^{3} \mathrm{erg} / \mathrm{cm}^{3}$ and $\mathrm{K}_{2}=$ $-3.0 \cdot 10^{3} \mathrm{erg} / \mathrm{cm}^{3}$. Once the anisotropy constants are known, we can calculate the effective anisotropy in the $x$ direction $(\theta=0)$ for small excursions from the $x$-direction. Equation 5 yields a field of 20 e along the long axis of our thin film. This field is small compared to the in-plane magnetostatic anisotropy field, which can be accessed for the centre region of a rectangular film. We calculated the demagnetisation factors: $N_{x}=0.0002 ; N_{y}=0.0009$; $\mathrm{N}_{\mathrm{z}}=0.9989$. This gives an magnetostatic anisotropy field of $4 \pi$ $M_{s} \cdot\left[N_{y}-N_{x}\right]=70 e$. As $K_{1}$ and $K_{2}$ represent all anisotropies due to the properties of the thin film, we can conclude that except the magnetostatic anisotropy, other anisotropy effects play a dominant role, such as field induced anisotropy or magneto elastic anisotropy at the edges of the thin film. The determination of the exact nature of the anisotropy requires further investigations.

Now, the most important parameter for our measurements and simulations of the LLG equation is the magnetic field pulse. We estimated this pulse in our waveguide by fitting it to the dynamics of the system at different bias fields. The leading edge of the pulse can be represented by a time $\tau_{\mathrm{r}}$, the rise time. For the form of the field pulse we chose:

$$
h(t)=h_{0}\left(1-\exp \left(-t / \tau_{r}\right)\right)^{3} \exp \left(-t / \tau_{f}\right)
$$

where $\tau_{f}$ denotes the decay time of the pulse and describes the trailing edge.

The decay time $\tau_{f}$ was obtained by fitting the pulse to the change of precession frequency in time (c.f. fig. 7). This fit also provides directly the strength of the field pulse and the saturation magnetisation of the film, which has also been measured and confirmed by VSM measurements. The determined decay time of the pulse lies between 500ps and 700ps. $h_{0}$ and $M_{s}$ were determined to be $(9 \pm 1)$ Oe and $(800 \pm 20) \mathrm{emu} / \mathrm{cm}^{3}$ respectively. Figure 9 shows the fits for bias fields of $460 \mathrm{e}, 940 \mathrm{Oe}$ and $1900 \mathrm{e}$ 
with the parameters $M_{s}$ and $h_{0}$ obtained from the fit in fig.7. We can conclude that the rise time of the pulse lies between 10ps and 60 s. For all simulations presented in this paper, we used a 90e field pulse with a rise time of $30 \mathrm{ps}$ and a decay time of $600 \mathrm{ps}$. Using these parameters the fits in figs. 7 and 9 are in good agreement with the measured values. Further more precise measurements will be performed by sampling the shape of the field pulse $\mathrm{s}^{3.57}$. Note that the fits carried out here are for the polar component of the magnetisation. For large bias fields the change in $\mathbf{M}_{\mathrm{x}}$ (the longitudinal component) is small and therefore the contributing longitudinal Kerr signal can be neglected compared to the polar signal. Figure 10 shows the bias field dependence of the precession frequency. From eqn. 3 it follows that the square of the precession frequency depends linear on the applied bias field. We simulated this dependence, with $\mathrm{M}_{\mathrm{s}}=800 \mathrm{emu} / \mathrm{cm}^{3}$ and $\mathrm{H}_{\mathrm{sx}}=$ 20e. The simulation is in good agreement with the measured values. Only at low bias fields the line deviates from the data. The reason for this discrepancy is due to the relatively larger field pulse strength as compared to the bias and the anisotropy field. As shown, the field pulse is added to the bias field and the vector sum will increase the precession frequency. For large bias fields this effect is small. For low bias fields, i.e. below $15 \mathrm{Oe}$, the field pulse of about $90 \mathrm{e}$ approximately adds $30 \mathrm{e}$ to the total field, which in first order approximation increases the precession frequency by $3 \%$.

Furthermore, a higher magnetostatic anisotropy field in the comer regions of the thin film element may influence the system, since we assumed the anisotropy to be constant over the hole film element. A larger magnetostatic anisotropy in the corner regions, which is reasonable for a flat rectangular film may also result in an increase of the precession frequency (c.f. eqn. 3). Only if the bias field is large enough, the film is magnetised uniformly and our assumption of constant magnetisation is valid.

\section{Conclusion}

We have shown that our technique, using a photoswitch in combination with a coplanar waveguide is a very good tool to study the spin dynamics in a weak ferromagnetic system. The device produces short in-plane field pulses of an amplitude, large enough to initiate a coherent precession of the NiFe (81:19) thin film system. We could estimate the field pulses to have a rise time of 10-60ps, a decay time of 500-700ps and a field strength of $90 \mathrm{e}$ at the peak. Furthermore the field pulses are short enough to enable the study of the dynamics of the system due to its magnetic properties. We have also shown that the anisotropy plays an important role on the precession of the system. The observation of slightly increased precession frequencies at low bias fields may also be due to the influence of a non-uniform anisotropy on the magnetisation dynamics. This has also been reported by other groups $^{1,2)}$. In order to quantify this effect a comparison to the response of an elliptical system with uniform magnetostatic anisotropy is needed.

\section{Acknowledgements}

The authors wish to thank for the good collaboration with all members of ZTMF1 at Siemens and for the helpful guidance, preparing the devices. This work was part of the research programme of the Stichting voor Fundamenteel Onderzoek der Materie (FOM) and financially supported by the Nederlandse organisatie voor Wetenschappelijk Onderzoek (NOW) and partly supported by the TMR network NOMOKE and the Brite Euram project Tunnel Sense.

\section{References}

1) T. J. Silva, T. M. Crawford: IEEE Trans. Magn., 35, 671 (1999)

2) W. K. Hiebert, A. Stankiewicz, M. R. Freeman: Phys.Rev.Letters, 79, 1134 (1997)

3) M.R. Freeman et al: IEEE Trans. Magn., 27, 4840 (1991)

4) D. H. Auston: IEEE J. of Quantum Electr., 19, 639 (1983)

5) U.D. Keil, D. R. Dykaar, A. F. Levi, R. F. Kopf, L. N. Pfeiffer, S. B. Darak, K. W. West: IEEE J. of quantum Electr., 28, 2333 (1992)

6) U.D. Keil: IEEE J. of quantum Electr, 32, 1664 (1996)

7) M. Y. Frankel, S. Gupta, J. A. Valdmanis, G. A. Mourou IEEE Trans. Microwave Theory Technol., 39, 910 (1991)

8) U. D. Keil, H. J. Gerritsen, J. E. M. Haverkort, H. J. Wolter: Appl. Phys. Lett, 66, 1629 (1994)

9) M. Bauer, R. Lopusnik, J. Fassbender, B. Hillebrands: Appl. Phys. Lett. 76, 2758 (2000)

10) T.M. Crawford, C. T. Rogers, T. J. Silva, Y. K. Kim, IEEE Trans.Magn, 32, 4087 (1996) 